\title{
ATTENDING TO THE AFFECTIVE: EXPLORING FIRST YEAR STUDENTS' EMOTIONAL EXPERIENCES AT UNIVERSITY
}

\author{
S. Bharuthram \\ Department of English \\ University of the Western Cape \\ Cape Town, South Africa \\ e-mail: sbharuthram@uwc.ac.za
}

\section{ABSTRACT}

This study engaged students at the affective level in order to acquire a better understanding of their emotional experiences at university with the ultimate aim of improving teaching and learning. A qualitative research study was undertaken whereby students wrote about their feelings at that particular moment, on two different occasions in a semester. The data reveals that students used mostly negative descriptors to express their emotions some of which included feelings of selfdoubt, alienation, loss of identity and not belonging to the university and their disciplinary community. The feelings expressed by students show that their emotions constitute the very core of their being and are therefore intertwined with the way they perceive their studies and themselves as students and as individuals. Hence, it is argued that emotional factors cannot be disentangled from teaching and learning and that spaces need to therefore be created in the curriculum for the inclusion of the affective domain.

Keywords: emotions, higher education, affective factors, teaching and learning

\section{INTRODUCTION}

Students enter higher education with a range of different life experiences and may therefore experience academia very differently one to another. Upon entering university, in addition to grappling with new interpersonal, financial and other issues, students are expected to take on a set of literacy practices or 'ways of being' which are often not open to critique or made overt to them (Boughey and McKenna 2016). This may result in different emotional responses related to classroom cultures, homework exercises, group work, attending classes, and writing tests and or examinations. These affective factors are generally not acknowledged or considered in the classroom by academics who generally view the curriculum as the 'business of the day' and disentangled from emotions (Zembylas 2012). However, underestimating the role of the affective in higher education in no way diminishes its effects as is evident in the recent student protests in South African higher education through which students have been demanding a more inclusive education that legitimizes their being, claiming that institutional cultures are stifling 
and alienating. Hence, it becomes imperative that universities and academics take cognisance of student emotions.

Consequently, this article attempts to address the affective domain by exploring its place in teaching and learning. With this in mind the author engaged first year students at the affective level with the purpose of acquiring an understanding of their emotional experiences that may either impede or enhance learning at university. The questions addressed in this research are:

- What do students' emotions tell us about them as students?

- Should there a place in the curriculum for helping students make sense of their emotional experiences and the implications thereof?

It is hoped that this work will contribute to those beginning to consider the role of the affective at university level (Francis and Le Roux 2011; Bozalek and Leibowitz 2012). It is also hoped that this research will lead to continued conversations on emotions and discomforting pedagogies in the classroom, ultimately leading to the re-visioning of the role of emotions in teaching and learning in academia.

\section{UNDERSTANDING EMOTIONS IN TEACHING AND LEARNING}

According to Trigwell, Ellis and Han (2012, 812), cognition, motivation and emotion are generally recognised as being '3 fundamental classes of human mental operations'. Yet, the focus in higher education is mainly on cognition and motivation despite the fact that emotions are unavoidable in academic settings and are considered an integral part of the educational process and of life more generally (Dirkx 2008). The importance of emotion is succinctly captured by Solomon $(2008,1)$ who states 'we live our lives through emotions, and it is emotions that give our lives meaning'. While some educators view the expression of emotions in the classroom setting as necessary and see it as a way to let students give vent to their feelings, after which students could refocus, many educators generally frown upon the display of emotions and view them as being 'obstacles to reason and the development of knowledge' (Dirkx 2008, 11) and as something that should be avoided in formal learning spaces.

However, in recent years more academics have begun to acknowledge the role of emotions in the classroom and more emphasis has been placed on the interrelationship of emotions and learning (see for example, Jarvis 2006). Many researchers have shown the positive effects of considering emotions in classroom settings (Pekrun et al. 2010; Pekrun and Linnenbrink-Garcia 2012). For example, Pekrun and Linnenbrink-Garcia (2012) found in their study on academic 
emotions and student engagement that emotions can have an influence on students' engagement; can re-inforce the storage and retrieval of information; and can increase motivational and behavioural engagement, which in turn will have a positive effect on academic learning and achievement. In this article the terms emotions, moods, feelings, and affect are used interchangeably as is often the case in educational discourse (see for example, Dirkx 2008; Beard, Humberstone and Clayton 2014).

An understanding of what emotions are is not straightforward as it varies according to the theory being drawn upon, and at times with variations existing even within theories. For example, some theorists view emotions as innate and inherent physiological responses to stimuli arising from the lower parts of the brain (Jarvis 2006). Cognitive theorists view emotions as physiological responses to external stimuli that are dependent on the individual's judgement and evaluation of situations (Dirkx 2008). Very different from the cognitive view is the social-constructivist view that sees emotions as social constructions that are context dependent. In this view emotional responses are learned or acquired and constructed over time within particular social and cultural contexts.

Situated within the socio-cultural perspective of learning is Lave and Wenger's (1991) theory of social practice in which 'learning' is viewed as a participation in social practice subjective as well as objective - whereby newcomers to a particular community of practice are both absorbing, and being absorbed in, the 'culture of the practice' (Christie et al. 2007, 6). However, studies drawing on Lave and Wenger's theory show that difficulties may arise in the process of becoming members of a community of practice if emotional dimensions come into play. Christie et al. $(2007,8)$ argue that '[i]t is the "culture shock” on entering higher education - the anxiety about not knowing what is expected or not understanding the new rules of the game - which underpins and becomes entangled with the learning process’.

A more recent view is the idea of emotions as embodied. This view originates from and is central to the idea of embodied knowing and learning - a more holistic approach - whereby ‘embodied learning has a strong emotional or feeling dimension’ (Merriam, Caffarella and Baumgartner 2007, 194). According to Varela, Thompson and Rosch $(1991,149)$ embodied learning and knowing represents a theory of knowledge production that 'depends on being in a world that is inseparable from our bodies, our language, and our social history'. These recent views, i.e. the constructive and holistic approaches, pose a challenge to the dominant understanding that learning relies exclusively on reason and objective scientific ways of knowing (Dirkx 2008, 15).

Much of the literature on emotions in teaching and learning has been contentious and 
sporadic. Furthermore, emotion in learning is often under- or mis-theorised (Beard, Clegg and Smith 2007, 236). According to Mortiboys (2002, 7), much of the culture in higher education tends to imply that universities are 'emotion-free zones' and there is a tendency to downplay the affective domains of learning as emotions are considered private and should be controlled; as such a display of emotions is regarded as irrational and disruptive. This conventional and westernised approach views emotion as the opposite of reason and the assumption is that the 'truth could only be reached when humans were completely devoid of subjectivity and emotions’ (Zembylas 2012, 4).

This line of thinking leads to what Zembylas (2012) refers to as the employability discourse, where a role of the higher education practitioner is to teach students personal emotional management skills that will enable them to 'fit in' with society and to function appropriately outside academia and more specifically in the workplace. As such, if students learn to control their emotions they may even become successful in their employment. In contrast, students who do show emotions in the classroom are perceived as having a 'low selfesteem' and are generally seen as 'troubled' students who need support in the form of therapy. Zembylas (2012) refers to this view as the therapeutic discourse. He argues that both these views (employability and therapeutic) are problematic as they suggest that individuals are separate from their emotions and do not take into account the individual as a 'whole person'. In considering the individual as a 'whole person', theorists who oppose the above views argue that social integration which includes the affective dimensions of student engagement with higher education is an important aspect in their success (Tinto 1994; Johnston 1997). To this end, Beard, Clegg and Smith $(2005,236)$ are of the view that the 'affective and embodied self are already aspects of all pedagogical encounters' and should therefore be taken cognisance of.

\section{THE CASE STUDY}

This research engaged students at the affective level in order to acquire a better understanding of their emotional experiences and feelings at university with the ultimate aim of improving teaching and learning by making it more 'holistic'. The participants were all first year undergraduate students from the Faculty of Community and Health Sciences (CHS) at a South African university, who were registered for an Academic Literacy module called English for Educational Development (EED). This course is part of the University's wider social justice project and the purpose of the EED module is to develop students' disciplinary literacy practices by making these explicit, thereby enabling greater epistemological access to disciplinary ways of knowing and doing as well as the institutional culture. In doing so the historical, social and 
political nature of practices is acknowledged and made accessible to students; however, often the focus tends to be on the discipline itself rather than the student. In other words, the affective domain is easily overlooked. Hence, I took a decision to include an affective component which can be viewed as a 'baby step' towards engaging in more extensive discussions on the role of emotions in teaching and learning.

The study was qualitative in nature and involved students writing about what they were feeling on two separate occasions in the first semester of the 2016 academic year. Since this research did not attempt to test a hypothesis, an inductive approach was used which allowed theory to be derived from the analysis of the data (Crossman 2007). The first writing task (Task 1) occurred in the first term on 8 March 2016 and which fell at the start of the 4th week of lectures. A total of 63 responses were collected. The second writing task (Task 2) occurred in term 2 on 19 April 2016 - approximately 6 weeks after the first writing task and a total of 60 responses were collected. Both tasks were given to students at the start of the lecture period and students were asked to write on a blank sheet for approximately 15 to 20 minutes reflecting on their current emotions - i.e. how they were feeling at that particular moment. This 'free writing style' allowed for free expression and thick description of their emotional experiences. The same task was given twice in order to get a sense of whether students' experiences had changed during the course of the semester. Altogether a total of 123 writing pieces were collected. However, after Task 1 and Task 2 were paired, there was a total of 51 students who participated in both the tasks. The balance, i.e. 21 written pieces, were ignored as some students had done Task 1 and not Task 2 and vice versa.

The research had the necessary ethical clearance from the institutional ethics committee. Ethical permission was also obtained from students who signed a consent form giving permission for their work to be used for research. The form explained that their participation in this study was entirely voluntary, that the EED course is research driven, that their writing tasks would be used for research purposes, and that their names would not be revealed in any analysis or publications arising from such analysis. Furthermore, they could at any time during the semester withdraw their participation in the research.

I analysed the two writing tasks using an interpretive method of coding (Erickson 1986) to ascertain students' emotional experiences. Each writing task was analysed separately after being collected. Once the analysis of both the tasks was completed, the two writing pieces were then paired and re-examined. Hence, each writing task was analysed twice. The pairing of the tasks enabled me to make comparisons of students' expressions of their feelings at two different times in the semester. 
The data was analysed and interpreted in line with the overall aim of the research and in particular, the first research question. Hence, during the first analysis of each writing task the keywords used by students to express how they were feeling were highlighted, for example, stressed, happy, frustrated, etc. These were then reflected graphically in a spider diagram which was further developed by listing the reasons provided by students on why they experienced a particular feeling, for example, feeling stressed because of not knowing how to answer an essay question. From these reasons clear themes began to emerge, for example, in relation to social relationships, financial issues, health issues, academic issues, etc. An analysis of these themes describes what students were feeling when they completed the writing task and is presented below. Notably, there were clear overlaps in the themes which represented all the categories of the life world (for a discussion on the categories see Beard Clegg and Smith 2007). Hence, rather than being presented by headings they are presented in a holistic manner. Similar methods of data analysis have been used by other researchers (see for example, Crossman 2007; Zembylas and McGlynn 2010).

\section{FINDINGS}

An analysis of the data revealed that students described their emotions in both Task 1 and Task 2 mostly through the use of negative descriptors. Selected exemplars are presented below in detail using direct quotations to capture the intensity of students' feelings, followed by a discussion of the data.

\section{Writing Task 1}

Most of students' concerns related to the academic project. Students felt very pressurised, overwhelmed and stressed because of the heavy workload and the limited time they had to complete tasks. This dominated their feelings: 'I am feeling extremely stressed and fairly exhausted. I feel as if everything is just piling (workwise) and I have no time in which to complete these said tasks - even more finding time for myself' (5). These feelings led to students doubting themselves and feeling inadequate which affected their sense of self. Some students used metaphors to express how they felt: 'I feel like I'm being slowly, but surely killed by all the work being thrown on my shoulder. It feels like I'm drowning in a pool full of work where there is so much to do, in such a short time. I feel scared and stressed ...' (31). These metaphors were powerful in communicating the student's perspective. Crossman (2007, 319) is of the view that metaphors and images 'appear to serve as dramatizations of emotional experience so that the listener is drawn to understand, engage in and empathize with the 
particular emotion described'. In some cases, consistent thoughts about the immense workload affected students' ability to focus on lectures: 'I can hardly focus on lectures thinking about what I need to do next' (49). In light of the heavy workload some students referred to the 'fast pace of university life' and expressed concerns keeping up. To this end, there were some students who blamed the lecturers for being inconsiderate, having 'unrealistic expectations' (42) and 'piling them with too much of work' (42) hence calling into question the role of the academic in understanding students’ perspectives which Rogers (1983) argues helps to improve the quality of learning.

Some students acknowledged the responsibility that comes with being a university student: 'I guess growing up comes with its load of work. I have to get used to making hard decisions and making sacrifices because I cannot have bread with butter on both sides ...' (17), while others attributed their feelings to a matter of adjusting to university life which they acknowledged is very different from school. Many students held a different perception of university life prior to entry: 'From outside I thought varsity was all about nice things and being easy but I came to realise that it is not' (17). For these students the adjustment was much more difficult. Others attributed their inability to cope with the workload as a result of their own lack of understanding of the work being covered: 'I feel confused because some of the thing I don't understand' (15). A few students expressed the view that schools do not adequately prepare students for university life: ‘I don’t think high schools are preparing pupils for varsity life. What I need right now is self-motivation to be able to push even if I am feeling like I'm going to die or my mind is about to break' (22). This view concurs with research findings that report that South African schools do not adequately prepare students to meet the challenges of higher education (Ngwenya 2010; Ralfe and Baxen 2012).

Some of the above feelings expressed by students demotivated them, which in turn led to feelings of hopelessness and despair: 'Varsity life has thrown me into the deep end and I am struggling to stay afloat' (38); questioning of their own capabilities: '... I haven’t even started with some [tasks], now I am feeling overwhelmed, frustrated and stressed because I don't know how I will manage to do all the school work I have’ (26); and uncertainty regarding their career choice: ‘... I feel like I don’t know what to do regarding my course choice. I feel like I’ve chosen the wrong one. ... my gut feeling is telling me I shouldn't be doing this course and guilt is mixed with this ...' (11). Clearly these students lacked the sense of belonging within their disciplinary and the university community as they grappled with a learning environment that was new and unfamiliar to them.

It also appears that these negative feelings had an influence on some students' sense of 
self, self-worth and confidence. There were concerns about letting family members down and strong emotions of shame, guilt and of 'losing face' surfaced at the possibility of failure or dropping out of university and having to face family members. A few students expressed concerns relating to the uncertainty of the requirements of academic tasks and whether they were meeting lecturers'/university expectations: ‘... Not knowing whether your assignment is up to standard, whether you have learnt enough for your test make me stressed' (45); 'I have prepared well but I'm still unsure about the new testing method' (50). This uncertainty and/or lack of clear expectations about disciplinary practices can hinder students' learning experiences (Christie et al. 2007). When students enter university, they are expected to take on a set of literacy practices which are often not made overt to them and they therefore see these as an imposition on their identity leading to feelings of alienation within the disciplinary community (DeKadt and Mathonsi 2003; Mgqwashu 2009).

There were some references to personal issues that included students feeling unwell leading to a lack of concentration and tiredness; death of a family member and illness of a parent which led to feelings of sadness, lack of focus and waiting for the day to be over. A few students reported living away from home and missing family members, and requiring the physical presence of their family to help them overcome challenges. For some, living away from home meant now having to do things for themselves and adjusting to a new routine of cooking, cleaning, washing, etc. which they were not used to doing. While many of the students in this category felt alone there were a few who indicated that they have 'amazing friends' who 'always manage to lift me up when I'm feeling down and they serve as a great support structure' (32). One student mentioned difficulty arising because of lack of resources. She mentioned that lecturers expected them to complete assignments and submit them typed and she did not have a computer at home or the Internet and therefore could not conduct any research from home. Of note is that in South Africa many students come from poorly resourced and less literate home and school environments which can impact negatively on their successful integration into teaching and learning communities (Bharuthram and Clarence 2012).

The majority of the students indicated that the workload was impacting negatively on their social lives with some of them stating that they no longer have a social life: 'I cannot wrap my head around how things changed so quickly within myself - how I used to be able to balance my time ... I officially feel like my social life is non-existent and this state of confusion, conflict and disappointment is leading me on a slow path of destruction' (44).

In contrast to the negative experiences described above there were a handful of students who reported feeling positive, energised, and good about themselves: 'I feel fabulous and when 
I feel fabulous my mind becomes more healthy so does my body ...' (3). Some attributed these positive feelings to their tendency to always look on the brighter side of things and for a few students these good feelings made them 'feel encouraged to do better and become better' (12). This finding is consistent with research studies that show that positive emotions 'generate coping mechanisms, and produce more creative, resilient, socially integrated and healthy individuals’ (Beard, Humberstone and Clayton 2014, 631). Interestingly, 3 students stated that while they felt a sense of contentment, they felt annoyed that they had to write about their feelings and found it a stressful exercise. This shows that being emotionally contented does not necessarily prevent the stress of higher education expectations but can make it more possible to confront them.

\section{Writing Task 2}

By this time in the semester many students seemed more confident and more settled. However, for some students their feelings around academic issues, in particular, the heavy workload and insufficient time to complete tasks, were still at the forefront and seemed to have intensified for some. Hence, these students continued to express feelings of being overwhelmed, stressed, frustrated, tired, drained, pressurised, etc. Coupled with this was the understanding that university life is 'tough' (41) and they have to work much harder and be more committed than previously.

A few students reported that their academic performance on tests and assignments thus far was disappointing and they attributed the poor results to incorrect study techniques and poor time management skills. Much of students' focus and concern seemed to be around the upcoming examinations, which they felt were very close. From the responses it also seems many students had 'matured' and found coping mechanisms and therefore felt less stressed: 'I am setting realistic yet fairly challenging goals for myself and scheduling time for my tasks on a basis of which ones are important and which ones are simply urgent and not of much importance' (41). It could be that the negative emotions served as activating emotions that motivated students (Pekrun, Goetz and Titz 2002).

There were a few students, however, who could not seem to find ways to cope better and this led to feelings of disillusionment: 'I feel like I don’t care anymore' (14). Within this group some indicated that they had not fully adjusted: 'I feel like I have not yet been able to completely find my feet in University ...' (51). It also appears that students felt very alone in their struggles: 'I wonder all the time if I am currently the only student with anxiety and stress and if I should be taking pills. ... I don't know what to do anymore about the stress ...' (49). A few students 
indicated a preference not to deal with their strong negative academic emotions and/or pretend these emotions did not exist: 'All I can do at this moment is pretend to be okay but deep down, there is a lot of hidden mixed emotions' (45). From these responses it is clear that students felt emotionally isolated and lonely and this affected their confidence (Turner 2006).

Having become more familiar with the courses/subjects they were studying, students were now able to express preferences; for example, they now felt comfortable in some subjects and described these as enjoyable, but were less comfortable with others which they thought were more challenging. Group work was brought up by a few students who reported it as stressful as not all group members participated equally. According to Dirkx $(2008,9)$ 'differences among students regarding values or interests, such as how to best proceed with a group project often lead to feelings of anger or frustration, .... Such emotional expressions are often bounded by curricular contexts in which the disagreement surfaces, but they may create an affect that endures for the remainder of that particular setting or even beyond' which is clearly evident in the current study.

A few students expressed financial concerns. The student who in Task 1 mentioned a lack of resources re-iterated this point. Financial concerns also included no money to purchase stationary, no lunch money, and insufficient money for transport to get to and from university. These concerns led to feelings of depression, disillusionment, and worry. Interestingly, the reference to their lack of social life which was mentioned in almost all responses in Task 1 seemed to fade as this was hardly mentioned by students in Task 2.

\section{DISCUSSION}

\section{What do students' experiences tell us about them as students?}

The rich descriptors used by students to express how they were feeling show that their emotions are embedded in their makeup, constituting the very core of their being, and are intertwined with the way they perceive their studies and academia generally, as well as their selves as students and individuals. Therefore, it would be a grave injustice to classify their emotions as 'irrational' or 'inappropriate' and as being separate from the individual. Such thinking is akin to the model of the student as the decontextualised learner in which learning is constructed as culturally, socially and politically neutral (Boughey and McKenna 2015) and the student is considered in ways divorced from her social context (Boughey and McKenna 2016). In this way of thinking, success in higher education is viewed as a result of attributes that are 'inherent in, or lacking from, the individual' (Boughey and McKenna 2016, 1). However, the data shows 
that 'learning does not take place within the realm of individual cognition' (Christie et al. 2007, 5) and students' emotions cannot be separated from their 'whole being'; the body is a 'source of knowledge about one’s self and one’s relationship to the world' (Dirkx 2008, 15).

Generally, students enter higher education with mixed emotions ranging from pride and excitement that they are entering a new chapter in their lives coupled with anxiety and concern for what this unknown chapter may hold. While the expectation is that students will soon settle into the system, adjust and begin to function optimally, the data shows that this may not be necessarily the case. While a few students are able to make the transition from high school to university with relative ease, the majority of the student participants were unable to do so leading to feelings of anxiety, frustration, despair, self-doubt, shame, and low self-esteem. For these students their sense of being competent learners was disrupted because of their unfamiliarity with their new learning environment, which they found difficult to navigate (Christie et al. 2007). It could also be that these students' primary Discourses (ways of being and doing within their homes and communities) may not be congruent with the Discourses of the university (i.e. the secondary Discourses - ways of being and doing within the university community) (Gee, 1990). For these students then the acquisition of academic Discourses is quite a challenge and they may view them as 'alien and even incomprehensible' (Boughey and McKenna 2016, 4).

It seems that the view students held of university life prior to entry differed from their actual experiences at university, leading them to conclude that schools do not adequately prepare them for university life. This is an issue that has been of great concern for many South African higher education practitioners and has also been discussed in research findings (Ngwenya 2010; Ralfe and Baxen 2012). The students’ descriptions of their emotions also show their emotional vulnerability, and therefore they should be nurtured into the system. For many students the negative emotions became so much stronger as the year progressed that they began to question their sense of self and self-worth. From the expression of students' uncertainty and lack of knowledge about the 'ways of being' and 'ways of doing' at university it would appear that "academia consists of a "code" that students are expected to crack without anyone mentioning that it exists or explaining how it works' (Boughey and Mckenna 2016, 4). These uncertainties may have a negative impact on teaching and learning as they leave students feeling demotivated, disillusioned and unable to focus on lectures, thereby affecting their overall performance. Research studies have shown that emotions do have an influence on students' academic learning and achievement (Villavicencio and Bernardo 2013). For example, anxietyladen tasks may reduce participation while tasks that students perceive as more enjoyable and 
interesting may lead to greater student investment.

Furthermore, the loss of a full social life is seen as another impediment and adds to students' emotional load. The above findings are consistent with the results of a study conducted by Beard, Clegg and Smith $(2007,248)$ who found that in students' descriptions of their emotions 'all categories of the lifeworld were present' in relation to: the personal project, the academic project, temporality, spatiality, sociality, embodiment, and discourse. Finally, this case study, albeit involving a relatively small number of students, also tells us that first year students need a great deal of mentoring and care to make their academic journey a rich one which begs the next question: Should there be a place in the curriculum for helping students make sense of their emotional experiences and the implications thereof?

Traditionally, educators were taught that being professional meant distancing themselves from their students (Labaree 2000), as forming emotional attachments with students could result in conflicts of interest (DeLaine 2000). This line of thinking had implications for the delivery of content whereby material was presented in an 'objective' manner and learning was viewed as neutral. It also had implications for the dealing with the affective in the classroom in that should student emotions surface during teaching these were either ignored or the student was told to supress or control his/her emotions and remain objective. However, as discussed above, the data presented in this research suggests that emotions are intertwined with other aspects of the students being which attests to the view that emotion and learning are 'interrelated and interactive and interdependent' (Antonacopoulou and Gabriel 2001, 435). Hence, we need to begin to view students as social beings who have their own life histories (Boughey and Mckenna 2015) and their own emotions arising from their life histories and experiences. As such, it is important for educators to acknowledge and value the range of social and cultural experiences that students bring with them to the classroom.

Furthermore, for first year students, 'getting to know' a new community of practice is an emotional experience that may be exciting for some but could also lead to feelings of 'being alone' and excluded for others (Christie et al. 2007). Hence, the argument presented in this article is that practitioners should create space within the curriculum and teaching to help students make sense of their emotional experiences as it is through these spaces that students could be provided the opportunity to critique their own assumptions about themselves and others (Zembylas and McGlynn 2010). Hargreaves (2001, 1060) is of the view that good teachers are able to increase learning by creating ways to make 'emotional understanding possible'. Through these spaces, academics could assist students in acquiring language that assists them in engaging with their feelings (Beard, Clegg and Smith 2007) and in this way give 
them a voice which could lead to students' feeling a sense of legitimacy and empowerment. Furthermore, students may also come to realise that their feelings may impact on their overall engagement which may then in adversely impact their learning and achievement. This realisation could result in students using different approaches when dealing with their studies and in handling challenges. It therefore becomes essential for educators to understand 'how to communicate with students and form relationships with them' (Crossman 2007, 325), which is described as 'a crucial aspect of the teacher’s role’ (325).

With regards to the expressing of academic emotions, in a study conducted by Beard, Clegg and Smith $(2007,249)$ in which students were given the opportunity to consider their feelings, the student participants reported this experience as being positive as many of them felt that expressing their feelings helped them reflect on their own engagement with the course and in particular the challenges of the course. In addition, students' feelings of being alone may be reduced just by listening to other students express similar academic emotions to the ones they hold. The conscious awareness in students of their own emotions as well as those of others may lead to caring and empathy that extends beyond the classroom. Beard, Clegg and Smith (2007, 250) caution that some of the emotional states that students experience may lie outside of the pedagogic engagement. However, they argue that 'if students can recognize the ways in which these aspects of their lives impact on their engagement in pedagogical spaces, and if they have a language to think through them and describe them, it seems likely that students can develop a better understanding of the energies and challenges involved in coming to terms with studying'.

In creating spaces for the affective in the classroom caution needs to be exercised as doing so may have associated risks, and ethical issues also need to be considered. Firstly, creating space for the affective may cause discomfort for both the practitioner and the student alike. In the case of students, some discussions may impact different students differently and could be quite damaging for them with lasting effects. For example, Zembylas and McGlynn $(2010,15)$ report that in their research on discomforting pedagogies on social injustices, the participants found the activity uncomfortable and the effects were reported months later. Furthermore, students may feel uncomfortable and anxious during discussions and this may deter them from participating in further discussions or even lead to avoidance of classes. Additionally, the teacher may not be well equipped to manage and facilitate discussions that may arise around sensitive issues and this could lead to divisions within students in the class. However, these challenges should not be seen as major stumbling blocks as they can be overcome. 


\section{CONCLUSION}

In this study the affective domain was addressed by exploring students' emotions at 2 different stages in a semester. The data shows that being at university and becoming a university student is an 'intrinsically emotional process' (Christie et al. 2007, 3). The experiences students expressed signify real emotions and acknowledging these is significant for a more holistic understanding of learning, since in writing about their emotions students not only focussed on academic issues but also on personal and social issues. This broader focus reinforces the concept of the 'whole person', more specifically that students cannot be viewed as being separate from their life histories and emotions. Therefore, for effective teaching and learning to occur, all aspects of a student's being should to be considered. Hence, this article presents an argument for creating spaces within the mainstream curriculum to consider affective factors as it is in the disciplines that students learn what knowledge is and how knowledge is constructed.

However, some cautions are sounded. In order for academics to create spaces in their curriculum on the affective domain in rich and meaningful ways, such discussions should be included in professional development programmes for academics. University teaching and learning specialists could also play a pivotal role in this process, for example, in collaborating with lecturers on curricular changes even to the extent of team teaching or by being present during pedagogical activities that include affect, even just to lend support. Such changes may result in teaching and learning becoming more meaningful and possibly also move students from 'passive empathy’ (see Zembylas and McGlynn 2010) to taking on active responsibility and action to reduce injustices. Continued such engagements may ultimately result in transformation.

Finally, I recommend extending this study to include classroom discussions which would provide students with the opportunity to not only share their own feelings but also learn from each other on how to engage with these feelings and the challenges they experience. Bearing in mind that emotions change during the course of learning, further research in the form of a longitudinal study is worthy of consideration.

\section{REFERENCES}

Antonacopoulou, E. and Y. Gabriel. 2001. Emotion, learning and organizational change. Towards an integration of psychoanalytic and other perspectives. Journal of Organisational Change Management 14(5): 435-451.

Beard, C., S. Clegg and K. Smith. 2007. Acknowledging the affective in higher education. British Educational Research Journal 33(2): 235-252.

Beard, C., B. Humberstone and B. Clayton. 2014. Positive emotions: Passionate scholarship and student transformation. Teaching in Higher Education 19(6): 630-643. http://dx.doi.org/10/1080/ 13562517.2014.901050 
Bharuthram, S. and S. Clarence. 2012. Teaching reading as a disciplinary knowledge practice in higher education. South African Journal of Higher Education 29(2): 42-55.

Boughey, C. and S. McKenna. 2015. South Africa: Universities can’t just wash their hands of student failure. The Conversation, 28 May 2015. https://theconversation.com/universities-cant-just-washtheir-hands-of-student-failure-40664

Boughey, C. and S. McKenna. 2016. Academic literacy and the decontextualized learner. Critical Studies in Teaching and Learning 4(2): 1-9.

Bozalek, V. and B. Leibowitz. 2012. An evaluation framework for a socially just institution. Higher Education for the public good: Views from the South, ed. B. Leibowitz, 59-72. Trentham \& SUN MeDIA: Stellenbosch, South Africa.

Christie, H., L. Tett, V. E. Cree, J. Hounsell and V. McCune. 2007. A real rollercoaster of confidence and emotions: Learning to be a university student. Online papers archived by the Institute of Geography, School of Geosciences, University of Edinburgh.

Crossman, J. 2007. The role of relationships and emotions in student perceptions of learning and assessment. Higher Education Research and Development 26(3): 313-327.

DeKadt, E. and N. Mathonsi. 2003. Writing in English with an 'African voice': Ownership, identity and learning. Journal of Language Teaching 37(1): 92-103.

DeLaine, M. 2000. Fieldwork participation and practice: Ethics and dilemmas in qualitative research. London: Sage Publications.

Dirkx, J. M. 2008. The meaning and role of emotions in adult learning. New Directions for Adult and Continuing Education 120: 7-18.

Erickson, F. 1986. Qualitative methods in research in teaching. In Handbook of research in teaching, ed. M. C. Wittrock, 11-161. New York: Macmillan.

Francis, D. and A. le Roux. 2011. Teaching for social justice education: The intersection between identity, critical agency, and social justice education. South African Journal of Education 31(3). http://www.scielo.org.za/scielo.php?script=sci_arttext\&pid=S0256-010020110003000

Gee, J. P. 1990. Social linguistics and literacies: Ideology in discourses. Basingstoke, Falmer.

Hargreaves, A. 2001. Emotional geographies of teaching. Teachers College Record 103: 1056-1080.

Jarvis, P. 2006. Toward a comprehensive theory of human learning. London: Routledge.

Johnston, V. 1997. Why do first year students fail to progress to the second year? An academic staff perspective. Paper presented at the British Educational Research Association Annual Conference, York, 11-14 September.

Labaree, D. 2000. On the nature of teaching and teacher education: Difficult practices that look easy. Journal of Teacher Education 51(3): 228-247.

Lave, J. and E. Wenger. 1991. Situated learning: Legitimate peripheral participation. Cambridge: Cambridge University Press.

Merriam, S. B., R. S. Caffarella and L. M. Baumgartner. 2007. Learning in adulthood: A comprehensive guide. San Francisco: Jossey-Bass.

Mgqwashu, E. 2009. On becoming literate in English: A during- and post-apartheid personal story. Language Learning Journal 37(3): 293-303.

Mortiboys, A. 2002. The emotionally intelligent lecturer. Birmingham, SEDA Publications.

Pekrun, R., T. Goetz and W. Titz. 2002. Academic emotions in students' self-regulated learning and achievement: A program of qualitative research. Educational Psychologist 37(2): 91-105.

Pekrun, R., T. Goetz, L. M. Daniels, R. H. Stupnisky and R. P. Perry. 2010. Boredom in achievement settings: Control-value antecedents and performance outcomes of a neglected emotion. Journal of Educational Psychology 102: 531-549.

Pekrun, R. and L. Linnenbrink-Garcia. 2012. Academic emotions and student engagement. Handbook of research on student engagement, ed. S. L. Christenson et al., 259-282. 
Ngwenya, T. 2010. Correlating first-year law students' profile with the language demands of their content subjects. Per Linguam 26: 74-99.

Ralfe, S. and J. Baxen. 2012. 'I don't like reading': The reading histories, identities and practices of first-year Bachelor of Education students. Conference presentation, HELTASA, Stellenbosch University, 28-30 November 2012.

Rogers, C. 1983. Freedom to learn for the 80s. Columbus, OH: Charles E. Merrill Publishers Co.

Solomon, R. C. 2008. True to our feelings: What emotions are really telling us. New York: Oxford University Press.

Tinto, V. 1994. Leaving college. Rethinking the causes and cures of student attrition. Chicago, IL, University of Chicago Press.

Trigwell, K., R. A. Ellis and F. Han. 2012. Relations between students' approaches to learning, experienced emotions and outcomes of learning. Studies in Higher Education 37(7): 811-824.

Turner, Y. 2006. Chinese students in UK business school: Hearing the student voice in reflective teaching and learning. Higher Education Quarterly 60(1): 27-51.

Varela, F., E. Thompson and E. Rosch. 1991. The embodied mind: Cognitive science and human experience. Cambridge, Mass: MIT Press.

Villavicencio, F. T. and A. B. I. Bernardo. 2013. Positive academic emotions moderate the relationship between self-regulation and academic achievement. British Journal of Educational Psychology 83: 329-340.

Zembylas, M. and C. McGlynn. 2010. Discomforting pedagogies: Emotional tensions, ethical dilemmas and transformative possibilities. British Educational Research, 1-19. First published on: 14 December 2010 (iFirst). doi:101080/01411926.2010.523779.

Zembylas, M. 2012. Reinstating or disrupting the dichotomy of reason/emotion in higher education? A historicized approach. Keynote to the Higher Education Close-up 6 Conference, Rhodes University, July 2012. 